\title{
Multi-Criteria Analysis for decision making applied to active distribution network planning
}

\author{
Gianni Celli ${ }^{\mathrm{a}}$, Nayeem Chowdhury ${ }^{\mathrm{a}}$, Fabrizio Pilo ${ }^{\mathrm{a}, *}$, Gian Giuseppe Soma ${ }^{\mathrm{a}}$, Matteo Troncia ${ }^{\mathrm{a}}$, \\ Iva Maria Gianinoni ${ }^{\mathrm{b}}$ \\ ${ }^{a}$ Department of Electrical \& Electronic Engineering - University of Cagliari, Piazza d'Armi, 09123 Cagliari, Italy \\ ${ }^{\mathrm{b}}$ Ricerca sul Sistema Energetico - RSE S.p.A., Via R. Rubattino 54, 20134 Milano, Italy
}

\section{A R T I C L E I N F O}

\section{Keywords:}

Distribution network planning

Distributed Energy Storage

Multi-Criteria Analysis

Analytic Hierarchy Process

\begin{abstract}
A B S T R A C T
Along with increasing presence of renewable energy in the distribution network, active network planning ap proach is of utmost interest for distribution system operators to harness the maximum benefits from the resources. However, it has been a challenge for the decision maker to choose the optimal planning option considering the multiple conflicting criteria. In this paper, a systematic and automatized approach for project selection based on Multi-Criteria Analysis is proposed for assessing a large set of planning alternatives. A case study has been done for a typical rural distribution network, a Pareto front of planning alternatives obtained by means of a multi-objective optimisation is analysed. Each alternative involves the optimal siting and sizing of storage units along with traditional network upgrading solutions. An automatized pairwise comparison procedure within the Analytic Hierarchy Process is proposed for rejecting subjectivity. The promising analysis illustrates the 'best' project selection considering nine criteria for storage system deployment to provide flexibility to the distribution network. The proposed approach aims at identifying the planning alternative that best satisfies the stakeholders' expectation considering the multiplicity of decision makers' points of view.
\end{abstract}

\section{Introduction}

Currently, the electrical power system is facing a paradigm shift led by different political, economic, and technical drivers. The liberalization of the electricity market and the penetration of the renewable energy sources changed the behaviour of the distribution network. Since the related capacity installed became significant in the distribution network, innovative approaches for planning and operation have become necessary. As highlighted in Refs. [1,2], the traditional fit and forget approach is no longer effective due to the opportunities from the active management of distribution network. Unlike the fit and forget approach which is based on traditional network reinforcement (e.g., building new lines and substations, and/or upgrading the existing ones), the active management approach involves the non-network solutions as development (e.g., generator dispatch, demand-side management, reactive power management, system reconfiguration, etc.). In this context, the maximum exploitation of the existing infrastructure can produce more cost-effective solutions if uncertainties are properly managed with probabilistic optimization for risk containment $[1,2]$.

Since the increased complexity, the modern distribution planning should be based on multi-objective approaches that are able to analyse, make compromises and select solutions among different alternatives $[1,2]$. In fact, distribution planning involves conflicting objectives such as maximize hosting capacity, reduce energy losses, improve service quality, reduce capital expenditure (CAPEX) and operational expenditure (OPEX). In literature, several optimization algorithms are used for solving the multi-objective problems; however, when multiobjective methods minimise an unique Objective Function (OF) obtained as the sum of multiple sub-objective functions they actually convert the multi-objective problem to a single objective [3]. This approach does not allow minimising/maximising the OFs independently; with the aim to find a number of optimal planning alternatives without introducing any a priori subjective preferences, a "true" multi-objective algorithm can be more effective [1]. The multi-objective a posteriori methods based on evolutionary algorithms have been widely studied for devising Active Distribution Network (ADN) planning alternatives [4-7]. The main advantage of multi-objective evolutionary algorithms used for network planning is the devising of a set of Pareto optimal alternatives of abstruse planning problems. However, when the Pareto front contains a large number of alternatives and/or the OFs are more

\footnotetext{
* Corresponding author.

E-mail address: pilo@diee.unica.it (F. Pilo).
} 
than two, then it is difficult to accomplish a simple decision-making process [1-3]. In this context arises the main drawback of multi-objective planning, identify the best planning option became tremendously difficult for decision makers. In order to outclass this shortcoming, a systematic and automatized analysis of the Pareto front is necessary.

With the aim to identify the planning solution that best fits with the decision maker's point of view, the use of Multi-Attribute Decision Making (MADM) technique has been introduced in Literature [8-11]. In Ref. [8] a fuzzy Analytic Hierarchy Process (AHP) is used to rank a set of four smart grid planning alternatives. The case study concerns the siting and sizing of distributed generator in radial distribution feeders (IEEE 33-bus). A hierarchical structure of evaluation criteria is used, and criteria weights have been elicited according to the societal point of view by consulting a pool of experts. They proved that Multi-Criteria Analysis (MCA) is highly successful in the evaluation of alternatives in the presence of heterogeneous criteria. The selection of the best distribution planning alternative among a set of 3 options is made by means of the TOPSIS technique in Ref. [9]. The assessment is made through 8 output-based evaluation criteria. Three weight schemes are considered with the aim to assess three different point of views. In Ref. [10] the planning project selection problem of a rural feeder is faced by combining the AHP and the VIKOR techniques. The hierarchical structure of the evaluation criteria encompasses 4 main areas: technical, environmental, social, and economic. Four categories of experts have been involved in the weighting process, 13 planning alternatives have been analysed. Moreover, AHP is used [11] to identify the appropriate location of smart metering in distribution feeders considering conflicting criteria such as network losses, voltage levels and reliability. Six possible planning alternatives have been analysed. The criteria weights have been elicited by experts from the utilities with the goal of satisfying investment policies.

As shown in Refs. [8-11], the MADM approach is suitable for supporting the project selection among a small set of feasible planning alternatives. In order to improve multi-objective planning process by outclassing its main drawback, an automatized MADM project selection method is proposed in this paper. The proposed project selection approach is then applied to a large set of optimal ADN planning options. The large set is the best-known Pareto front originated by a multi-objective optimization involving the optimal siting, sizing, and scheduling of Distributed Energy Storage (DES). The planning alternatives are compared by using an automatized pairwise comparison procedure that considers output-based performance indices. In order to obtain a robust result for all possible decision makers' point of view, the MADM evaluation is repeated and the outcomes combined. The proposed methodology for project selection can support the decision makers in analysing large sets of planning alternatives. Moreover, the presented formalisation aims at filling the gap in the multi-objective planning by means of an automatic analysis of the obtained outcome.

\section{Multi-Criteria Analysis for decision-making in planning}

Planning is a decision-making activity which requires the assessment of a set of feasible investment options for identifying the best one. Typically, the optimal solution has to achieve a comfortable level of performance on several conflicting criteria by minimizing the related cost. Since those goals can be mutually conflicting, the decision maker has to make trade-offs taking into account the stakeholder perspective. Traditionally, the planning options are assessed by means of economicbased tools (i.e., Cost-Benefit Analysis - CBA) which require the conversion of all project impacts in monetary terms. These methodologies are acknowledged tools for considering only costs and benefits that can be directly monetised. In contrast, the appraisal of projects which show wide range effects and/or non-negligible intangible impacts shows some underlying shortcomings related to techniques for quantifying, monetising, and discounting the impacts [12]. In this context, the project selection process is biased. In the planning processes, MCA has been introduced in several sectors (e.g., transportation and environment) to better consider sustainability aspects and improve the effectiveness of the process [13]. Similarly, smart grid planning is a complex task, it aims at achieving more goals than the cost minimisation and the enhanced reliability of supply. Even if services and impacts in power system have a related cost, the monetisation procedure may distort their actual relevance for the specific planning process. The need is for more transparent and objective output-based project selection approaches. Therefore, shifting from a traditional economic-based assessment to new assessment tools is recommended [1,14]. In Europe, several guidelines have been released with the aim to promote the use of a multi-criteria framework on smart grid project assessment [15-17]. Unlike CBA, MCA allows considering the impacts directly, the monetising procedure that introduces an undesired latent point of view is avoided. In addition, the uncertainties related to monetary conversion are prevented. Moreover, since MCA involves directly the stakeholder point of view, transparency on the project selection process is provided. In fact, the consequences of a change of the analysis perspective are clear, as the stakeholders' point of view influences only the evaluation criteria relevance and not also the impact metrics. Nevertheless, MCA and CBA are not conflicting tools, a joint use can combine their strengths by mutual compensating their respective weaknesses [13]. In fact, MCA lacks on imposing that overall benefits have to exceed costs therefore, unlike CBA, MCA may be unable to identify the most cost effective options [18].

MCA is a systematic approach helps the decision maker in finding the preferred solution. The scientific Literature proposed several methodologies that belong to MCA [18]. Among them, MADM methods are suitable for multi-criteria decision problems whose goal is to find the best alternative among an explicitly known set. Considering a planning process, multi-objective optimization and MADM are complementary: the former can be used for devising a set of Pareto optimal solution; while a MADM method can be used to identify the best alternative of the set according to the planner expectations. Key features of MADM methods are the Performance Matrix (PM), the hierarchy of evaluation criteria, and the preferences of the stakeholders [18]. The entries of the PM are the values of performance of the alternatives with respect to the evaluation criteria. In the scoring stage, the elements of the PM are normalized to a common numerical scale. The preferences of the stakeholders are used to define the relative importance of the evaluation criteria during the weighting stage, a numerical value is assigned to each criterion as a relative weight. Basically, an MADM method is an algorithm that assesses the given alternatives on the basis of the PM and weights of criteria. The output of the algorithm is a ranking of the alternatives, the alternative that dominates all the others is the best alternative according to the decision maker point of view.

\section{Formalisation of the proposed automatized MADM approach}

In this paper, the proposed MADM approach aims at finding the best alternative among a large set of options. The methodology presented is based on the AHP which is one of the most acknowledged MADM techniques [18]. Moreover, the proposed automatic pairwise procedure is inspired by the procedure in Ref. [19] which has been adapted and generalised for addressing any PM. The scientific novelty of the proposed methodology is the formalisation of an assessment procedure for completing a multi-objective ADN planning approach for a given MV network. Furthermore, in order to identify a robust result, the presented approach considers all possible stakeholder scenarios modelled by different criteria weight schemes. Even if a Pareto front is studied in this paper, the proposed assessment approach can be used for any appraisal of large initial set of planning alternatives.

The MCA methodology proposed in this paper can be resumed in 3 key steps. 
Table 1

Saaty's judgment scale [20].

\begin{tabular}{ll}
\hline Verbal judgement & Saaty's scale $\left(\mathrm{w}_{\mathrm{j}} / \mathrm{w}_{\mathrm{k}}\right)$ \\
\hline Indifference/equal preference & 1 \\
Weak preference for object $\mathrm{w}_{\mathrm{j}}$ & 3 \\
Strong preference for object $\mathrm{w}_{\mathrm{j}}$ & 5 \\
Demonstrated preference for object $\mathrm{w}_{\mathrm{j}}$ & 7 \\
Absolute preference for object $\mathrm{w}_{\mathrm{j}}$ & 9 \\
\hline
\end{tabular}

1. Automatized scoring stage.

2. Overall score evaluation for each possible weight scheme.

3. The final score evaluation for each alternative.

The alternative that achieves the highest final score is acknowledged as the best of the set under analysis.

\subsection{The automatized scoring stage}

In authentic AHP, the subjectivity of the decision maker influences both the scoring and weighting stage. In the scoring stage, the decision maker has to express his preference on the alternatives by means of a pairwise comparison process. The personal judgment of the decision maker is quantified on a standardized judgment scale (Saaty's scale), as shown in Table 1.

Verbal judgement on object $w_{k}$ with respect to object $w_{j}$ are expressed by reciprocal numerical values.

In this paper, to undertake a scoring stage without introducing subjectivity, an automatic pairwise comparison process for avoiding the decision maker involvement is proposed. The automatic scoring procedure in Ref. [19] has been modified and generalised. The steps of the proposed automatic pairwise comparison procedure are represented in the flux diagram in Fig. 1.

Firstly, for each k-th criterion is evaluated a "direct ratio" preference matrix $Q^{(k)}$ whose entries are $q_{i, j}^{(k)}=a_{i}^{(k)} / a_{j}^{(k)}$, where $a_{i}^{(k)}$ and $a_{j}^{(k)}$ are, respectively, the performances of the $i$-th and the $j$-th alternative on the $k$-th criterion. According to the straightforward use of the Saaty's ratio scale, the value of $q_{i, j}^{(k)}$ expresses how much the i-th alternative is preferred than the $\mathrm{j}$-th one. With the aim to exploit the AHP methodology for the analysis, the obtained $q_{i, j}^{(k)}$ values have to be converted in terms of the Saaty's scale. Therefore, the scaling function $S$ in (1) is employed (1).

$r_{i, j}^{(k)}=S\left(q_{i, j}^{(k)}\right)=\operatorname{round}\left(1+\frac{\left(R_{\max }-R_{\min }\right) \cdot\left(q_{i, j}^{(k)}-1\right)}{q_{\max }^{(k)}-1}\right)$

where $r_{i, j}^{(k)}$ is the image of the element $q_{i, j}^{(k)}$ in the new scale, $q_{\max }^{(k)}$ is the maximum value among all the $q_{i, j}^{(k)} . R_{\max }$ and $R_{\min }$ are the maximum and the minimum value of the preferences in the destination scale; if the destination scale is the Saaty's ratio scale: $R_{\max }=9$ and $R_{\min }=1$. If all performance values of the alternatives are different from zero, the value of $q_{\max }^{(k)}$ is finite thus the scaling function $S$ can be applied. Conversely, if at least one alternative has a performance equal to zero, the value of $q_{i, j}^{(k)}$ is a division by zero. In this case, if $q_{i, j}^{(k)}$ is evaluated as a mathematical limit, the value of $q_{\max }^{(k)}$ tends to infinity. In addition to the model in Ref. [19], to avoid this event without losing the generality of the scaling process, the proposed automatic pairwise comparison algorithm finds $q_{h i g h}^{(k)}$ which is the highest $q_{i, j}^{(k)}$ less than infinite. Once the $q_{\max }^{(k)}$ value is obtained, the scaling function $S$ is applied to the modified "direct ratio" preference matrix $Q^{(k)}$.

Finally, the preference matrix $R^{(k)}$ related to the $k$-th criterion is obtained. Then, the consistency of the obtained preference matrices $R$ is verified. The proposed MADM approach is devised for analysing large

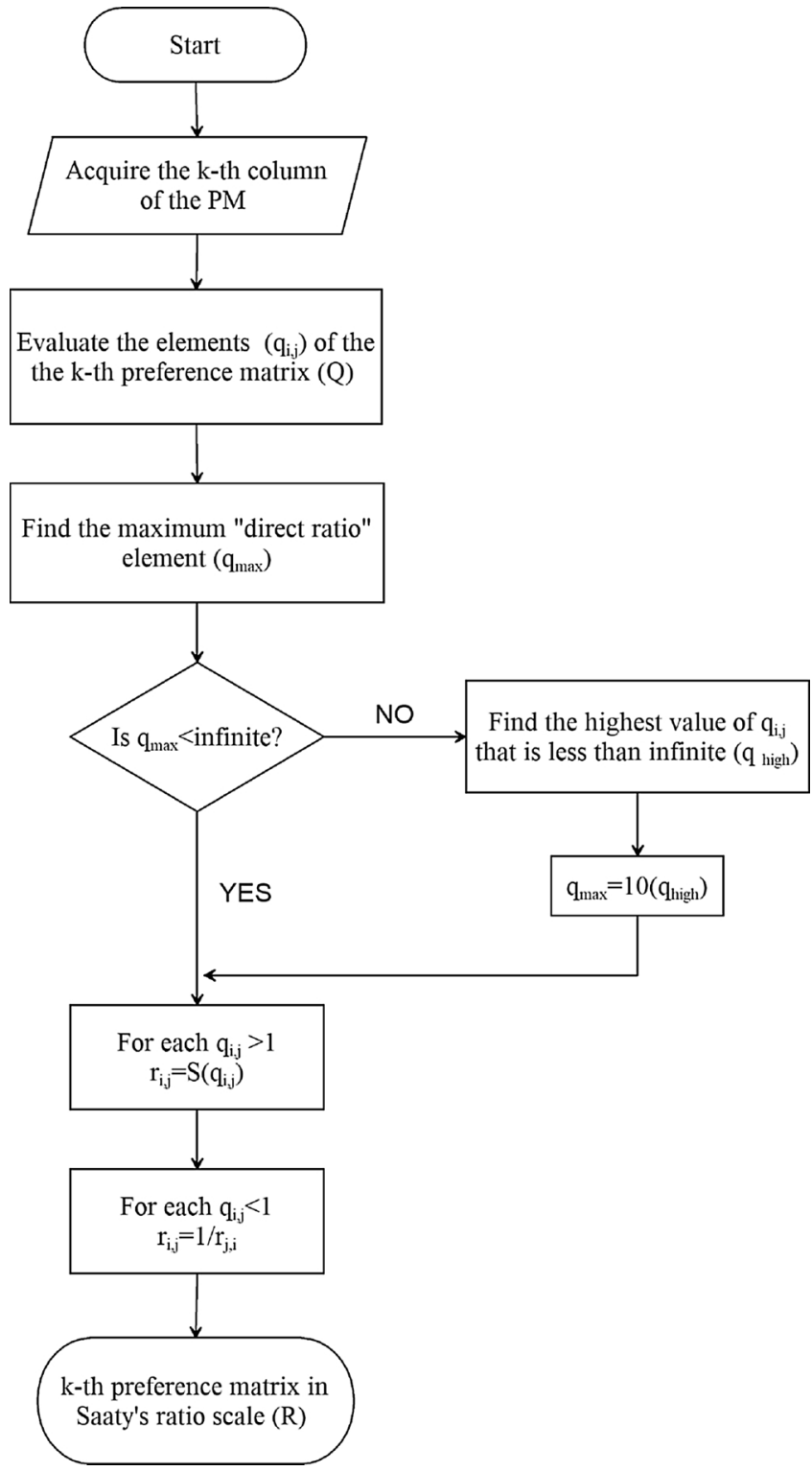

Fig. 1. Flowchart of the automatic pairwise comparison algorithm.

set of alternatives, hence the statistical method presented in Ref. [21] is exploited.

In the last step, the priorities (or normalised scores) of each preference matrix are evaluated. Priorities from preference matrices are evaluated according to authentic AHP: priorities are equal to the normalized eigenvector of the maximum eigenvalue of the preference matrix.

\subsection{The overall score evaluation}

Once that the normalised score of the alternatives is evaluated on all criteria, the overall score of each alternative is obtained by means of (2).

$O S_{i}=\sum_{j=1}^{n_{c}} p_{i, j} \cdot v_{j}$

where $O S_{i}$ is the overall score of the $i$-th alternative; $p_{i, j}$ is the normalised partial score of the $i$-th alternative with respect to the $j$-th criterion; $v_{j}$ is the global priority of the $j$-th criterion; $n_{c}$ is the number of the terminal criteria of the hierarchy. The alternative that achieves the 
highest overall score $O S$ is the one that the AHP indicates as the best alternative of the analysed set. The overall scores obtained by means of (2) are related to a single decision maker's point of view which is accounted as evaluation criteria relevance modelled by the assigned relative weights. Thereby, a different decision maker's point of view is modelled by different weights, hence the obtained ranking of alternatives may change. In order to find a robust result, in this paper an approach for accounting all possible points of view of the decision makers is proposed. The aim is to identify the alternative which is identified as the best considering all possible criteria weights values. To assess all perspectives of the decision maker, the proposed approach repeats (2) for each pattern of weight that respects (3).

$\left\{\begin{array}{c}\sum_{k=1}^{n_{c}} v_{k}=1 \\ 0 \leq v_{k} \leq 1\end{array}\right.$

where $v_{k}$ is the weight of the $\mathrm{k}$-th criterion.

\subsection{The final score evaluation}

From each evaluation of (2), the higher overall score and the label of the related alternative being collected. This final score is calculated as the sum of the overall score obtained by the best alternative on each AHP run, as shown in (4).

$F S_{i}=\sum_{j=1}^{n_{\text {best }, i}} O S_{i, j}$

where $F S_{i}$ is the final score of the i-th alternative, $O S_{i, j}$ is the overall score of the $\mathrm{i}$-th alternative obtained in the $\mathrm{j}$-th AHP evaluation, $n_{\text {best }, i}$ is the number of the AHP evaluations in which the i-th alternatives obtained the highest overall score. Namely, for each alternative, its final score is related to the sum of the overall scores obtained only when it results as the best of the set for a particular criteria weight scheme. This approach allows to emphasise the option that is labelled as best more times and collects the highest overall scores. The planning option which achieves the highest final score is identified as the best of the set under analysis.

\section{Case study}

The decision-making problem addressed in this paper is formalised by means of a flat structure. The hierarchy consists of three layers: the main goal, the evaluation criteria, and the design options under analysis. The alternatives under analysis belong to a Pareto front provided as output from a multi-objective planning optimisation. In order to undertake an output-based analysis, the evaluation criteria chosen for the MADM assessment match with the objectives of the multi-objective planning; therefore, the performance indicators of the alternatives are evaluated as the objective function values. As a consequence, these values are the entries of the PM.

\subsection{The grid under analysis}

The ADN planning alternatives regard a typical scenario of a real distribution network located in a rural area (Fig. 2). The studied network is weakly meshed with emergency tie connections, and radially operated [22]. Two primary substations feed 22 MV nodes ( 9 trunk nodes and 13 lateral nodes) that deliver power to MV and LV customers. The urban area (zone A1) consists of two underground feeders $\left(95 \mathrm{~mm}^{2}\right.$ MV underground cables are used). The zone B1 is rural area fed by an overhead feeder and characterized by the presence of a photovoltaic generators and a passive lateral branch (zone $\mathrm{C} 1$ ). The second rural area (zone B2) shows a high density of distributed generation. In the rural areas, trunk feeders and lateral branches are made by overhead lines with sections of $35 \mathrm{~mm}^{2}$ and $16 \mathrm{~mm}^{2}$ respectively.

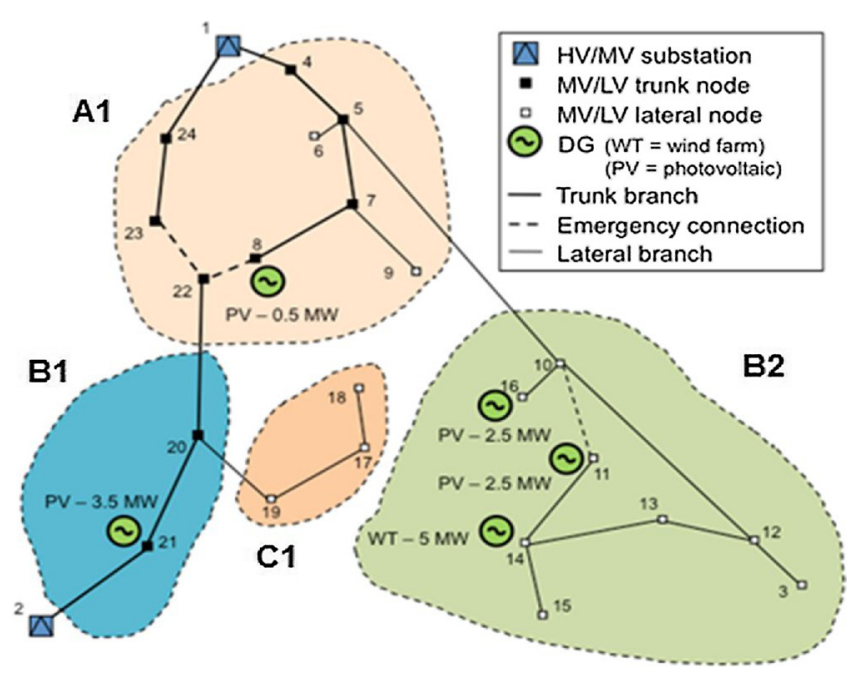

Fig. 2. Distribution network of the case study [22].

\subsection{The multi-objective optimisation planning framework}

Since the scope of this paper is to introduce an MCA based approach for distribution network planning project selection, the procedure used for obtaining the set of planning alternatives is not discussed in detail. The multi-objective optimization planning that devised the Pareto front under analysis has been made according to the procedure described in Ref. [23]. For the sake of consistency, only general aspects of the planning framework are described in this paper.

The duration of planning study is 10 years long that is a medium to long term planning that involve new primary and secondary substations. The topology of the MV network is fixed for the whole planning period. For each MV/LV node, a constant load growth rate of $3 \%$ per year has been assumed. Each load and generator have been modelled with typical daily profiles that take into account the uncertainties by a normal probabilistic distribution function. Network calculations are based on a probabilistic load flow which evaluates for each hour of typical days the steady state and the emergency network configurations. The resulting probability distribution of nodal voltages and branch currents are used for checking the technical constraints violation risk. If the risk is considered not acceptable, several development plans based on a combination of network and non-network solutions are assessed. The devised planning options involve lines and substations upgrading (as traditional network solutions) and DES siting, sizing, and management (as no-network solution). Amongst the non-network planning solutions, DESs optimally allocated can provide to the Distribution System Operator (DSO) numerous technical and economic benefits such as voltage support, losses reductions, enhanced reliability and quality of service, improved hosting capacity, deferral of network investments, and OPEX reduction [24]. Those benefits are not mutually exclusive because a single storage device can be used to offer different services [24]. Therefore, to understand the multiplicity of benefits, it is obvious to analyse DESs planning alternatives trough a multi-criteria methodology. In this case study, Li-ion batteries have been employed as DES technology, with a lifespan of 10 years, a nominal power range of $100 \mathrm{~kW} \div 3 \mathrm{MW}$, and a nominal duration from 1 to $10 \mathrm{~h}$. The unitary costs of related to this technology are $200 € / \mathrm{kW}$ and $400 € / \mathrm{kWh}$. All nodes have been considered as eligible for hosting a DES device, each planning option can host from 0 to 2 devices. The present study considered the same scenario as in Ref. [23], i.e., the DSO has the ownership of DESs which is used for network operation, energy price arbitrage is not allowed. An active control of loads and generators has not been involved as planning solution. The baseline scenario considered for planning does not employ DES devices, therefore it only considers traditional network solutions for facing network issues. 
When the whole network configuration satisfies the technical compliance in the planning horizon, the solution is memorised in the iterative planning process.

\subsection{The impact metrics, the objective functions}

All nine objectives used in the multi-objective optimisation are considered as criteria for the MADM assessments with the aim to emphasise the differences among the planning alternatives. To provide an overview on the impact metrics, the formulas of the objective function are described in this section. The aim of decision-making problem is to identify the best planning option; to this end, the black start support criterion is considered fulfilled by the alternatives which have a higher value of the related index, while the remaining eight criteria are fulfilled by the alternatives which exhibit lower values in the related objective functions.

\subsubsection{Network investment}

The network investment objective evaluates the economic value of the traditional network investment. This cost, $C_{i n v}$, encompasses only the investment in new/upgraded lines and substations, is evaluated by (5).

$C_{i n v}=\sum_{j=1}^{N_{\text {branches }}} C_{0 j}=\sum_{j=1}^{N_{\text {branches }}}\left(B_{0 j}+M_{0 j}-R_{0 j}\right)$

where $N_{\text {branches }}$ is the number of network branches, $C_{0 j}$ is the present cost of the $j$ th branch, and $B_{0 j}, M_{0 j}$, and $R_{0 j}$ are respectively it is building, management and residual costs actualised at the beginning of the planning period by considering a prefixed discount rate.

\subsubsection{Energy losses}

The Joule energy loss for the $j$ th branch in the $k$ th sub-period through is evaluated according to (6).

$E_{L j k}=8760 \cdot 3 \cdot R_{j} \cdot N_{k} \cdot\left(I^{2}{ }_{f j k}+I^{2}{ }_{0 j k}+I_{f j k} \cdot I_{0 j k}\right)$

where $I_{0 j k}$ and $I_{f j k}$ are the branch current at the beginning and at the end of the sub-period respectively, $N_{k}$ is the sub-period duration in years, and $R_{j}$ is the branch resistance. 8760 is the number of hours per year, 3 is the number of conductors. The total energy losses, $E_{L}$, are then obtained by summing the contributions of all branches in each sub-period.

\subsubsection{Reactive power exchange with the Transmission System Operator}

The interface inverter of DES devices can be used to separate the exchange of active and reactive power. This aspect can be used to manage the flows of reactive power in the high voltage side of the primary substations and to limit the costs that the distributors have to pay. In this paper, that cost is calculated according to Italian regulation framework (resolution 654/2015/R/EEL).

\subsubsection{Black start support}

DES devices can be used to run the black start of a share of the whole grid. The metric $P_{B S}$ of the black start support of each DES device consists in the sum of all the available power in each time slot. A greater $P_{B S}$ value implies a higher black start capability, it is evaluated as in (7).

$P_{B S}=\sum_{h=1}^{N_{h}} \min \left(\operatorname{SoC}_{h} \cdot \eta_{s c h}, P_{n}\right)$

where $S o C_{h}$ is the state of charge in the $h$-th time slot, $N_{h}$ is the number of the time slots, $P_{n}$ is the nominal power rate of the storage device, whereas $\eta_{s c h}$ is the discharging efficiency.

\subsubsection{Cost of the Energy Storage System}

The cost of the DESs is evaluated by their CAPEX related to voltage installation in the planning period. The CAPEX is evaluated as by means of (8).
$C_{C A P E X}^{D E S}=k_{p c s} \cdot c_{p} \cdot P_{n}+c_{e} \cdot P_{n} \cdot d_{n}$

where $k_{p c s}$ is the oversizing factor of the power conversion system, $P_{n}$ is the nominal power rating of the device, $d_{n}$ is the nominal duration. $c_{p}$ and $c_{e}$ are the specific costs, their value is respectively $200 € / \mathrm{kW}$ and $400 € / \mathrm{kWh}$. The residual value is assessed by considering a lifetime of 10 years. The maintenance cost and the OPEX related to the charge/ discharge losses has been disregarded.

\subsubsection{Quality of service - duration of interruptions}

The quality of service guaranteed by each planning alternative depends on the duration of the interruptions that each end-user observes. This impact is measured by the System Average Interruption Duration Index (SAIDI), which is calculated as in (9).

$S A I D I=\frac{\sum_{i=1}^{n} U_{i} \cdot N C_{i}}{\sum_{i=1}^{n} N C_{i}}$

where $N C_{i}$ is the number of customers in the $\mathrm{i}$-th bus, $U_{i}$ is the annual outage for customers in the $i$-th bus, whereas $n$ is the overall number of busses of the network. The aim of this objective is to evaluate the improvement of the reliability offered by the DES system. In fact, thanks to islanding operation it is possible to avoid the outage effects to the set of costumers involved.

\subsubsection{Quality of service - frequency of interruptions}

The quality of service also is measured by the System Average Interruption Frequency Index (SAIFI), which is calculated as in (10).

$S A I F I=\frac{\sum_{i=1}^{n} \lambda_{i} \cdot N C_{i}}{\sum_{i=1}^{n} N C_{i}}$

where $\lambda_{i}$ is the failure rate, $N C_{i}$ is the number of customers in the $i$-th bus, whereas $n$ is the overall number of busses of the network.

\subsubsection{Voltage regulation}

DES devices can contribute to voltage regulation with suitable injections of reactive and active power. The metric for the voltage regulation objective measures the difference between the maximum and the minimum bus voltage in the last year of the planning period. It is evaluated as in (11).

$r e g V_{\text {index }}=\sum_{i=1}^{N_{\text {nodes }}} \sum_{h=1}^{N_{f}}\left|V_{\text {max. } i}^{h}-V_{\text {min. } .}^{h}\right|$

$V_{\max . i}^{h}$ and $V_{\min . i}^{h}$ are the maximum and minimum voltage of the i-th bus in the h-th time interval. $N_{\text {nodes }}$ is the overall number of buses whereas $N_{f}$ is the sub-period duration used for the evaluation of the daily profile of loads and generators. To avoid double counting, this objective measures the contribution of DES to the quality of voltage; the contribution on avoiding voltage constraints violation is already accounted as deferral of network upgrading investment.

\subsubsection{Quality of services - voltage dips}

DES can improve the quality of service by decreasing voltage dips occurrence and transient interruptions caused by short-circuits. The metric of the voltage dips objective measures the annual cumulative frequency of the voltage dips considering all the busses of the MV grid.

\subsection{The Pareto front, the set of planning alternatives}

Each element of the obtained Pareto front is a different active planning non-dominated solution that involves the use of DESs. Each alternative is different by another for the number, the position in the grid, the power rating, and the daily energy scheduling of the DES devices, as well as for the traditional network upgrading solutions deployed. Since the obtained Pareto front consists of 1200 different 


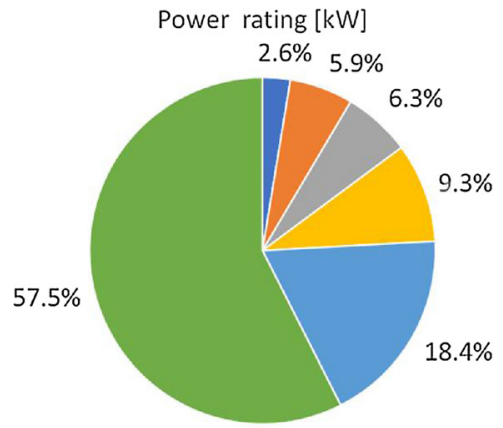

— 100-500-600-1000 - $=1100-1500$

$\square 1600-2000=2100-2500=2600-3000$

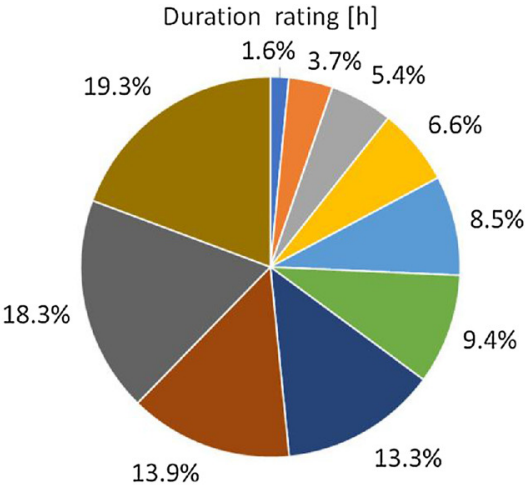

$\square 1 \square 2 \square 3 \square 4 \square 5 \square 6 \square 7 \square 8 \square 9 \square 10$

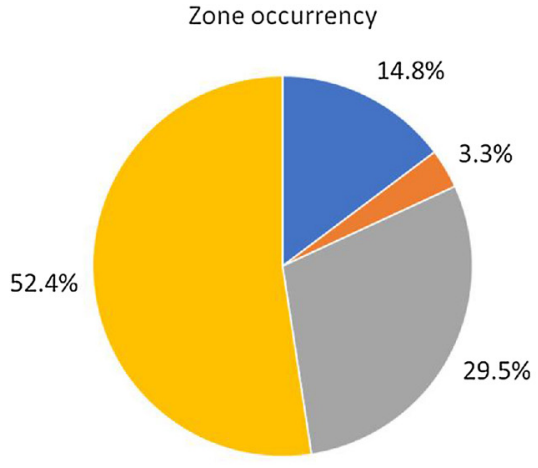

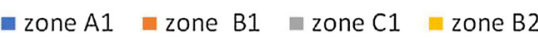

Fig. 3. Occurrence of DES devices characteristics among the planning options.

planning alternatives, only a general description of the set is given in this paper. Each planning alternative in the Pareto front involves 2 DES devices at most. 1042 alternatives are characterised by 2 DES devices, 157 involves one only device, the baseline alternative has no DES installed. In Fig. 3 the occurrence of DES devices in terms of power rating, duration, and on the installed busses considering all the alternatives of the Pareto front is provided.

Since the Pareto front contains the alternatives under analysis, it defines the PM which is the input of the MADM method. The PM has $\mathrm{N}_{\mathrm{a}}$ rows and $N_{c}$ columns; where $N_{a}$ is the number of the planning alternatives, while $\mathrm{N}_{\mathrm{c}}$ is the number of the criteria. In the present case study, the chosen evaluation criteria are the 9 objectives of the multi-objective planning; therefore, the Pareto front feature values are the entries of the PM; hence: $N_{a}=1200$ and $\mathrm{N}_{\mathrm{c}}=9$. Due to the large number of alternatives, the full PM is not reported in this paper. However, the performances of the alternatives on the 9 criteria are depicted in Fig. 4 in relative terms with respect to the baseline scenario. For each objective function, the difference between the performance value of each alternative and the baseline scenario has been calculated. Then, the obtained figures have been scaled according to the maximum absolute difference value. For the sake of clarity, the minimum and the maximum values of performance on each criterion are resumed in Table 2, along with the objective function values of the baseline scenario.

\subsection{The automatic MADM assessment}

Once the PM is built, the automatic MADM assessment follows the procedure described in Section 3. The automatic scoring algorithm produces $\mathrm{N}_{\mathrm{c}}=9$ squared preference matrices whose dimension is
Table 2

Minimum and maximum values of performances in the PM.

\begin{tabular}{|c|c|c|c|c|}
\hline Index & & $\begin{array}{l}\text { Minimum } \\
\text { value }\end{array}$ & $\begin{array}{l}\text { Maximum } \\
\text { value }\end{array}$ & $\begin{array}{l}\text { Baseline } \\
\text { scenario }\end{array}$ \\
\hline Network costs & {$[\mathrm{k} €]$} & 2174.2 & 3208.2 & 2205.0 \\
\hline Energy losses & [MWh] & 9664.2 & 32732.6 & 11216.1 \\
\hline $\begin{array}{c}\text { Reactive power } \\
\text { exchange }\end{array}$ & {$[\mathrm{k} €]$} & 0 & 43282.0 & 973.9 \\
\hline Black start & [MW] & 144.00 & 0 & 0 \\
\hline DES cost & {$[\mathrm{k} €]$} & 0 & 115200.0 & 0 \\
\hline SAIDI & [h/year] & 1.630 & 2.026 & 2.026 \\
\hline SAIFI & [occurrences/year] & 0.735 & 0.837 & 0.837 \\
\hline $\begin{array}{l}\text { Voltage } \\
\text { regulation } \\
\text { index }\end{array}$ & [p.u.] & 5.258 & 11.636 & 11.483 \\
\hline $\begin{array}{l}\text { Voltage dips } \\
\text { index }\end{array}$ & [Vdips/year] & 83.22 & 100.43 & 100.43 \\
\hline
\end{tabular}

$\mathrm{N}_{\mathrm{a}}=1200$. The obtained preference matrices are consistent, the related Consistency Ratio is less than 0.03. The priorities of the alternatives are evaluated from each preference matrix. The calculation (2) for obtaining the overall scores has been repeated for each weight scheme defined by (3). A step size of $\Delta v_{k}=0.05$ is considered among each evaluation point. The final score of the alternative is evaluated as defined by (4).

\section{Results and discussion}

Tables 3-5 present the results of the MADM evaluations. Only the top 5 alternatives are described. In Table 3 the overall scores obtained

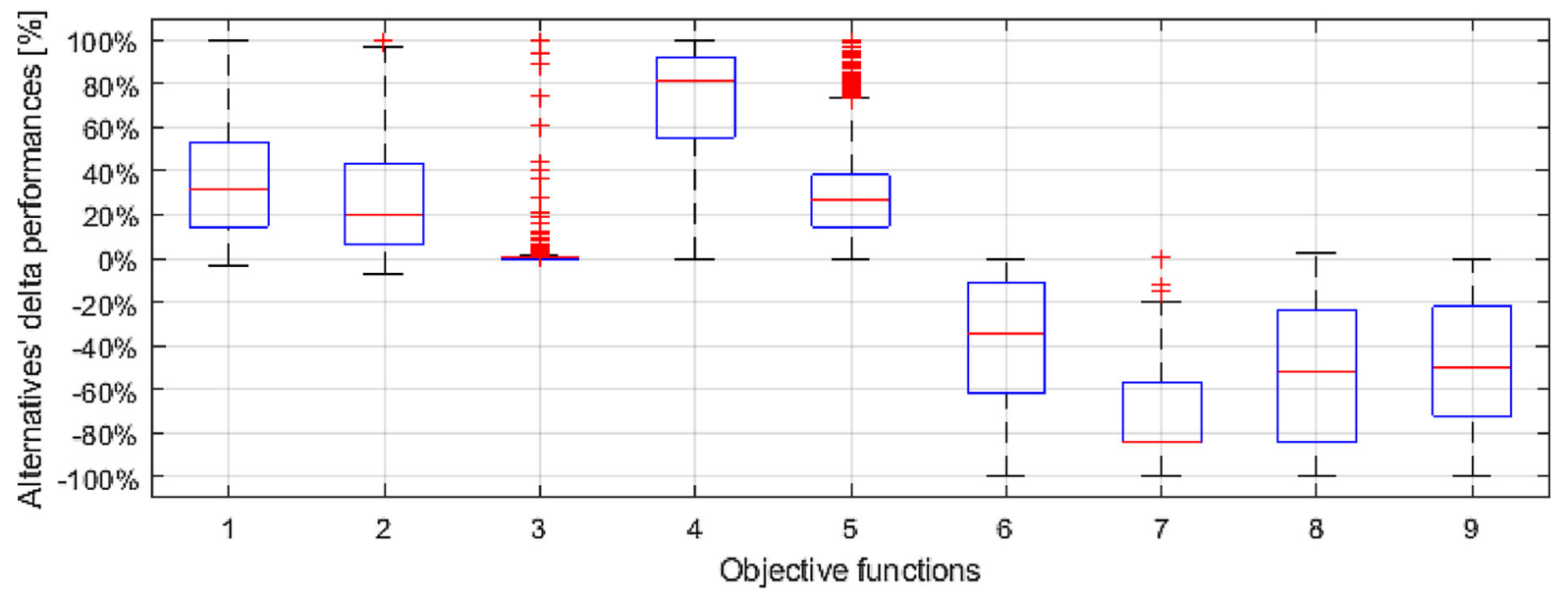

Fig. 4. Relative performances of the alternatives with respect to the baseline scenario. 
Table 3

Final scores.

\begin{tabular}{llllll}
\hline Alternative & A1069 & A4 & A110 & A113 & A477 \\
\hline Final score & 460.8 & 282.1 & 158.8 & 22.8 & 14.1 \\
Hits & $1,321,101$ & 815,250 & 610,899 & 96,334 & 56,003 \\
\hline
\end{tabular}

after all the evaluations are shown (normalised in thousandths). In addition, the row "Hits" represents how many times the corresponding alternative has been pointed out as the best considering all pattern of weights. Table 4 shows the PM of the top 5 alternatives. The left pie chart in Fig. 5 represents the distribution in relative terms of final score values among the alternatives; similarly, the right pie chart in Fig. 5 resumes the distribution of "hits" obtained by the alternative on each AHP assessment. Table 5 presents the DES topological information of each top 5 alternatives. As highlighted in Table 3 and Fig. 5, the alternative A1069 achieves the highest final score and is labelled as best most of times, therefore is the best alternative identified by the method. Fig. 5 shows that the first three alternatives obtain a final score higher than the sum of the final score of the remaining alternatives. This result is obtained also by considering the first position distribution depicted in the right pie chart. Accordingly, the alternatives A1069, A4, and A110 outclass all remaining alternatives belonging to the Pareto front.

As shown in Fig. 5, the overall performance achieved by A1069 is significantly higher than the remaining four top alternatives, hence the result obtained is considered robust. Among all 1200 analysed alternatives, 857 alternatives obtained a final score greater than zero; while 343 alternatives have not been identified as best alternative for any of the considered weight scheme. Considering the whole Pareto front, A1069 shows the highest performances on criteria such as voltage dips, reactive power exchange, and black start support. In addition, on SAIDI, SAIFI, and voltage regulation index A1069 exhibits great performances. The performance of A1069 for network investment and energy loss lies between the best and worst alternatives on these criteria. Even though the DES cost related to A1069 is the highest among all top 5 alternatives, it is still less than the half of the highest value observed in the Pareto front (115.2 M€). Therefore, A1069 can be considered as the best compromise design option. Along with an average expenditure of DES, it has achieved an overall superiority among all the criteria. The second-best alternative proposed by the evaluation is the baseline scenario (A4). As it can be seen in Table 4, A4 has the worst performances in five criteria, while it performs extremely well on the other four criterions. Due to criteria relevance in some AHP evaluation, the best alternative has been A4 when weights of these 4 criteria were higher. Namely, the output provided highlights the compensative peculiarity of AHP.

Except for A4 (no DES), all the alternatives ranked in the top 5 positions encompass siting of DESs in the network zone B2. It confirms that storage is more effective in reducing interruptions and enhancing voltage quality if placed in the rural areas with high distributed generation density. Besides these positive aspects, an increased level of losses and network investment is expected in the distribution network. Table 4 shows that A1069 involves a share of network investments and energy losses of $21.3 \%$ and $73.1 \%$ respectively, greater than the
Table 5

DES data of the first five alternatives ranked by AHP.

\begin{tabular}{|c|c|c|c|c|c|c|}
\hline \multirow{2}{*}{$\begin{array}{l}\text { Individual } \\
\text { A1069 }\end{array}$} & \multicolumn{2}{|l|}{ DES bus } & \multicolumn{2}{|c|}{ DES power size [kW] } & \multicolumn{2}{|c|}{ DES energy size [kWh] } \\
\hline & 10 & 12 & 3000 & 2900 & 30,000 & 20,300 \\
\hline A4 & No DES & No DES & - & - & - & - \\
\hline A110 & 3 & 12 & 2000 & 2400 & 18,000 & 14,400 \\
\hline A113 & 10 & 16 & 1600 & 2900 & 14,400 & 26,100 \\
\hline A477 & 3 & 12 & 1500 & 1700 & 300 & 15,300 \\
\hline
\end{tabular}

baseline scenario. The alternatives A110, A113, and A447 have an amount of DES CAPEX that is about $50-80 \%$ less than A1069. A lower level of DES CAPEX implies devices with less power and/or energy size. Consequently, the overall benefit achieved with these design options is lower. A1069 can be suitable for DSOs in a scenario where the regulatory framework allows them to focus more on quality of service than on costs (i.e., investments are fully refunded to DSO). On the contrary, an increased level of energy losses and of network investment can be undesirable for the decision maker. Prior to the MADM assessment, a subset of the Pareto front can be identified on the basis of the decision maker's threshold levels (i.e., a budget cap, maximum and minimum levels on some criteria). For the sake of completeness, the alternatives A1069 and A110 are compared with the alternative A1 which final score is zero. The comparison is represented in Fig. 6 and it is made in relative terms with respect to the baseline scenario as in Fig. 4. A1 involves the busses 15 and 21 by installing two DES devices of $2.6 \mathrm{MW} \div 3 \mathrm{~h}$ and $2.7 \mathrm{MW} \div 2 \mathrm{~h}$, respectively. Although A1069 introduces more network cost, energy loss, and DES cost than A1, the latter has a slight negative impact in terms of reactive power exchange; no impact in terms of SAIDI, SAIFI, and voltage dips; a lower positive impact on black start support and voltage regulation indexes. Therefore, only on three criteria A1 has a better performance than the A1069. A110 performs better than A1069 on DES cost, SAIDI, SAIFI, and voltage regulation; it has an equal performance in terms of voltage dips; conversely, on the remaining 4 criteria A110 is outclassed by A1069.

\section{Conclusions}

In this paper, a systematic and structured approach for project selection of smart grid development has been presented. A full deployment of the proposed methodology allows for an optimal planning of distribution networks helping the decision maker in finding the design option that best fits with the stakeholders' expectation. By assessing all possible points of view the most supported planning option is found. The case study concerns the analysis a Pareto front of ADN design options devised by an a posteriori multi-objective algorithm. Among the optimal set, the best alternative is identified by the proposed automatic MADM evaluation. The non-network planning options have been devised concerning siting, sizing, and scheduling of DES devices. Even if the MADM technique upon which the proposed approach is based is well available in Literature, the proposed automatized version improves the state of the art of MADM and makes it more suitable to distribution planning in the era of smart grids: (i) it allows planners to easily examine large sets of planning options; (ii) it enhances the objectivity of

Table 4

Performance matrix of the first five alternatives ranked by AHP.

\begin{tabular}{|c|c|c|c|c|c|c|c|c|c|}
\hline Alternative label & $\begin{array}{l}\text { Network costs } \\
{[\mathrm{k} €]}\end{array}$ & $\begin{array}{l}\text { Energy losses } \\
\text { [MWh] }\end{array}$ & $\begin{array}{l}\text { Reactive power exchange } \\
{[\mathrm{k} €]}\end{array}$ & $\begin{array}{l}\text { Black start } \\
{[\mathrm{MW}]}\end{array}$ & $\begin{array}{l}\text { DES cost } \\
{[\mathrm{k} €]}\end{array}$ & $\begin{array}{l}\text { SAIDI } \\
{\left[\frac{\mathrm{h}}{\text { year }}\right]}\end{array}$ & $\begin{array}{l}\text { SAIFI } \\
{\left[\frac{\text { occ. }}{\text { year }}\right]}\end{array}$ & $\begin{array}{l}\text { Voltage regulation index } \\
\text { [pu] }\end{array}$ & $\begin{array}{l}\text { Voltage dips index } \\
{\left[\frac{\text { Vdips }}{\text { year }}\right]}\end{array}$ \\
\hline A1069 & 2676 & 19,415 & 0 & 141.6 & 48,965 & 1.814 & 0.751 & 6.022 & 83.22 \\
\hline A4 & 2205 & 11,216 & 974 & 0 & 0 & 2.026 & 0.837 & 11.483 & 100.43 \\
\hline A110 & 2821 & 19,357 & 1084 & 104 & 23,740 & 1.63 & 0.735 & 5.738 & 83.22 \\
\hline A113 & 2193 & 12,647 & 1054 & 106.3 & 25,020 & 1.863 & 0.751 & 10.934 & 83.22 \\
\hline A477 & 2508 & 13,917 & 976 & 68.9 & 9610 & 1.636 & 0.79 & 8.748 & 83.22 \\
\hline
\end{tabular}




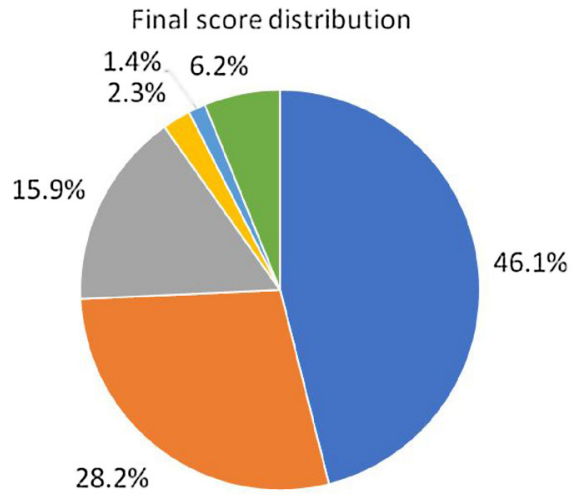

First position distribution

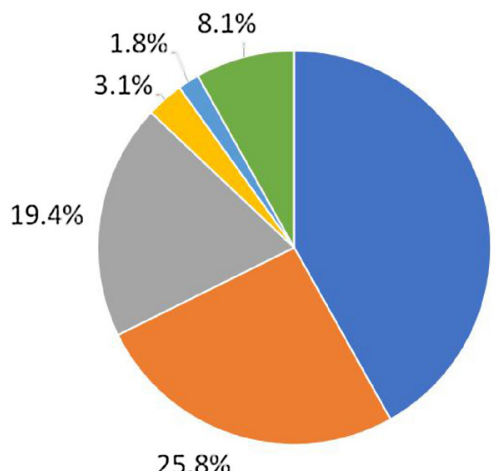

$41.9 \%$

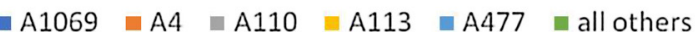

Fig. 5. Distribution of final scores (on the left) and of "hits" (on the right) obtained by the alternatives.

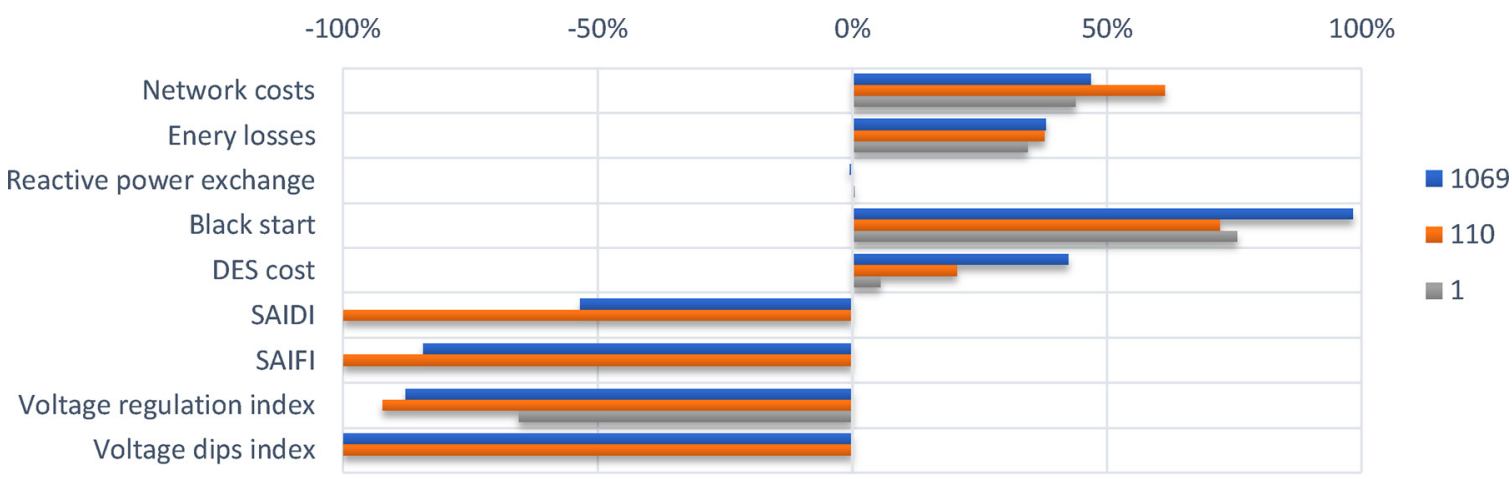

Fig. 6. Comparison of the performances of A1069, A110, and A1 in relative terms with respect to the baseline scenario.

the assessment; (iii) it is less biased than the CBA. Eventually, a robust planning option that considers the point of view of several stakeholders can be identified increasing the worth of multi-objective-attribute optimal planning. As inferable from the discussion of the results, comparing the alternatives is a laborious task, which complexity increases as the number of the alternatives and/or of the evaluation criteria increases. Therefore, an automatized tool which provides concise information about the best alternative in the set is fundamental for the decision-making processes.

\section{Acknowledgements}

Nayeem Chowdhury has been funded from the European Union's Horizon 2020 Research and Innovation Programme under Grant Agreement No. 676042. This work has been co-financed by the Research Fund for the Italian Electrical System under the Contract Agreement between RSE S.p.A. and the Ministry of Economic Development in compliance with the Decree of March 8, 2006, and by the Fondazione di Sardegna (project ODIS, CUP: F72F16003170002).

\section{References}

[1] G. Doorman, et al., Capacity mechanisms: needs, solutions and state of affairs, CIGRE Technical Brochure 647, February, 2016 p. 117.

[2] CIGRE WG C6.19, Planning and Optimization Methods for Active Distribution Systems, CIGRE, 2014.

[3] K. Miettinen, Introduction to multiobjective optimization: noninteractive approaches, Multiobjective Optimization, Springer, Berlin, Heidelberg, 2008, pp. $1-26$.

[4] A.V. Pombo, J. Murta-Pina, V.F. Pires, Distributed energy resources network connection considering reliability optimization using a NSGA-II algorithm, 2017 11th IEEE International Conference on Compatibility, Power Electronics and Power Engineering (CPE-POWERENG) (2017) 28-33.

[5] S.A.H.S. Olia, M. Jooshaki, M. Moeini-Aghtaie, M. Fotuhi-Firuzabad, Developing a multi-objective framework for planning studies of modern distribution networks,
2016 International Conference on Probabilistic Methods Applied to Power Systems (PMAPS) (2016) 1-6.

[6] M.G. Ippolito, M.L. Di Silvestre, E. Riva Sanseverino, G. Zizzo, G. Graditi, Multiobjective optimized management of electrical energy storage systems in an islanded network with renewable energy sources under different design scenarios, Energy 64 (January) (2014) 648-662.

[7] O.D. Montoya, A. Grajales, R.A. Hincapié, M. Granada, R.A. Gallego, Methodology for optimal distribution system planning considering automatic reclosers to improve reliability indices, 2014 IEEE PES Transmission Distribution Conference and Exposition - Latin America (PES T D-LA) (2014) 1-6.

[8] A. Janjic, S. Savic, L. Velimirovic, V. Nikolic, Renewable energy integration in smart grids-multicriteria assessment using the fuzzy analytical hierarchy process, Turk. J. Electr. Eng. Comput. Sci. 23 (November (6)) (2015) 1896-1912.

[9] İ. Şahin, M. Bağriyanık, Multiple criteria decision making in distribution system planning, 2015 9th International Conference on Electrical and Electronics Engineering (ELECO) (2015) 453-457.

[10] J.C. Rojas-Zerpa, J.M. Yusta, Application of multicriteria decision methods for electric supply planning in rural and remote areas, Renew. Sustain. Energy Rev. 52 (December (Suppl. C)) (2015) 557-571.

[11] R.G. Milbradt, L.N. Canha, P.B. Zorrilla, A.R. Abaide, P.R. Pereira, S.M. Schmaedecke, A multicriteria approach for meter placement in monitoring of smart distribution systems, Power Engineering Conference (UPEC), 2013 48th International Universities (2013) 1-6.

[12] F. Ackerman, L. Heinzerling, Pricing the priceless: cost-benefit analysis of environmental protection, Univ. Pa. Law Rev. 150 (May (5)) (2002) 1553.

[13] P. Beria, I. Maltese, I. Mariotti, Multicriteria versus cost benefit analysis: a comparative perspective in the assessment of sustainable mobility, Eur. Transp. Res. Rev. 4 (3) (2012) 137-152.

[14] D. Mills, L. Vlacic, I. Lowe, Improving electricity planning - use of a multicriteria decision making model, Int. Trans. Oper. Res. 3 (May (3)) (1996) 293.

[15] V. Giordano, I. Onyeji, G. Fulli, M. Jiménez, C. Filiou, Guidelines for conducting cost-benefit analysis on smart grid projects, Jt. Res. Cent. Ed. JRC Sci. Tech. Res. (2012) 72.

[16] V. Giordano, I. Onyeji, G. Fulli, M. Jiménez, C. Filiou, Guidelines for cost benefit analysis of smart metering deployment, Jt. Res. Cent. Ed. JRC Sci. Tech. Res. (2012) 88

[17] V. Giordano, S. Vitiello, J. Vasiljevska, Definition of an assessment framework for projects of common interest in the field of smart grids, Jt. Res. Cent. Ed (2014) 81 pages.

[18] J. Dodgson, M. Spackman, A. Pearman, L. Phillips, Multi-Criteria Analysis: A Manual, Department for Communities and Local Government, London, 2009.

[19] A. Mazza, G. Chicco, A. Russo, Optimal multi-objective distribution system 
reconfiguration with multi criteria decision making-based solution ranking and enhanced genetic operators, Int. J. Electr. Power Energy Syst. 54 (2014) 255-267.

[20] T.L. Saaty, A scaling method for priorities in hierarchical structures, J. Math Psychol. 15 (June (3)) (1977) 234-281.

[21] J.A. Alonso, M.T. Lamata, A statistical criterion of consistency in the analytic hierarchy process, Modeling Decisions for Artificial Intelligence (2005) 67-76.

[22] G.G. Soma, F. Pilo, G. Celli, Multi-objective programming for optimal WiMax allocation in smart grid, AEIT Annual Conference (2013) 1-5.

[23] G. Celli, F. Pilo, G. Pisano, G.G. Soma, Distribution energy storage investment prioritization with a real coded multi-objective Genetic Algorithm, Electr. Power Syst. Res. 163 (2018) 154-163.

[24] G. Celli, F. Pilo, G.G. Soma, D.D. Canto, E. Pasca, A. Quadrelli, Benefit assessment of energy storage for distribution network voltage regulation, CIRED 2012 Workshop: Integration of Renewables into the Distribution Grid (2012) 1-4. 